\title{
Rules of Competitive Stimulus Selection in a Cholinergic Isthmic Nucleus of the Owl Midbrain
}

\author{
Ali Asadollahi, Shreesh P. Mysore, and Eric I. Knudsen \\ Department of Neurobiology, School of Medicine, Stanford University, Stanford, California 94305
}

In a natural scene, multiple stimuli compete for the control of gaze direction and attention. The nucleus isthmi pars parvocellularis (Ipc) is a cholinergic, midbrain nucleus that is reciprocally interconnected to the optic tectum, a structure known to be involved in the control of gaze and attention. Previous research has shown that the responses of many Ipc units to a visual stimulus presented inside the classical receptive field (RF) can be powerfully inhibited when the strength of a distant, competing stimulus becomes the stronger stimulus. This study investigated further the nature of competitive interactions in the Ipc of owls by using two complementary protocols: in the first protocol, we measured the effects of a distant stimulus on responses to an RF stimulus located at different positions inside the RF; in the second protocol, we measured the effects of a distant stimulus on responses to RF stimuli of different strengths. The first protocol demonstrated that the effect of a competing stimulus is purely divisive: the competitor caused a proportional reduction in responses to the RF stimulus that did not alter either the location or sharpness of spatial tuning. The second protocol demonstrated that, for most units, the strength of this divisive inhibition is regulated powerfully by the relative strengths of the competing stimuli: inhibition was strong when the competitor was the stronger stimulus and weak when the competitor was the weaker stimulus. The data indicate that competitive interactions in the Ipc depend on feedback and a globally divisive inhibitory network.

\section{Introduction}

A midbrain network plays an essential role in selecting the next stimulus for gaze and attention (Carello and Krauzlis, 2004; McPeek and Keller, 2004; Fecteau and Munoz, 2006). The network consists of the optic tectum (OT), called the superior colliculus (SC) in mammals, and a group of nuclei in the lateral tegmentum referred to as the isthmic nuclei (Wang, 2003). Although this network exists in all vertebrate species, it is most highly differentiated anatomically in birds.

Each of the structures in the midbrain network represents the locations of stimuli topographically, as a retinotopic map of space. In birds, the nucleus isthmi pars parvocellularis (Ipc) is a cholinergic nucleus that receives precise topographic projections from the $\mathrm{OT}$ and returns equally precise topographic projections to the OT (Wang et al., 2006). Because of the reciprocal, point-topoint geometry of the interconnections, this circuit is thought to support space-specific enhancement of responses in the OT. The nucleus isthmi pars magnocellularis (Imc) is a GABAergic nucleus that receives a topographic projection from the OT and sends broad projections to all portions of the space map in both the Ipc and the OT, but not to the portion that provided input (Wang et al., 2004). This circuit is thought to mediate global

\footnotetext{
Received Jan. 3, 2011; revised Feb. 8, 2011; accepted Feb. 11, 2011.

Author contributions: A.A., S.P.M., and E.I.K. designed research; A.A. performed research; A.A. analyzed data; A.A. and E.I.K. wrote the paper.

This work was supported by National Institutes of Health Grant R01 EY019179 (E.I.K.). We thank P. Knudsen for technical support, and A. Goddard, S. Devarajan, C. Dunn, and A. Bryant for reviewing this manuscript.

Correspondence should be addressed to Ali Asadollahi at the above address. E-mail: asad@stanford.edu.

DOI:10.1523/JNEUROSCI.0023-11.2011

Copyright $\odot 2011$ the authors $\quad 0270-6474 / 11 / 316088-10 \$ 15.00 / 0$
}

competitive interactions across the maps of space within the midbrain network (Marín et al., 2007; Asadollahi et al., 2010; Mysore et al., 2010).

Previous research suggests that the Ipc participates in the selection of the most salient stimulus. Ipc units have well defined spatial receptive fields, they respond to multiple sensory modalities, they are not selective for stimulus features, and they respond with increasing firing rates to increasing contrast or motion (Maczko et al., 2006; Asadollahi et al., 2010). These properties resemble those of a salience map, a central component in computational models of stimulus selection and gaze control (Itti and Koch, 2001; Parkhurst et al., 2002). Equally important for competitive stimulus selection, Ipc units exhibit global competitive surrounds (Asadollahi et al., 2010): A stimulus located anywhere outside of the classical receptive field (RF) suppresses responses to another stimulus located inside the RF, and the amount of suppression depends on the relative strengths of the two stimuli.

What are the rules of competitive stimulus selection in the Ipc? A previous study demonstrated that the responses of Ipc units to a stimulus inside the RF can, for many units, be abruptly and powerfully suppressed, when the strength of a competing stimulus exceeds the strength of the RF stimulus (Asadollahi et al., 2010). This switch-like change in inhibitory strength that depends on the relative strengths of competing stimuli indicates that inhibition in the Ipc is regulated in an unusual way. This study introduces two additional protocols that characterize these competitive interactions more completely. The results reveal rules of competitive inhibition that are consistent with the hypothesis that the Ipc contributes to the selection of the most salient stimulus. 


\section{Materials and Methods}

\section{Animal and surgery}

We used nine adult barn owls (Tyto alba) of either sex in this study. All procedures were approved by the Stanford University Institutional Animal Care and Use Committee and were in agreement with the National Institutes of Health Guide for the Care and Use of Laboratory Animals and the guidelines of the Society for Neuroscience.

Animals were anesthetized with isoflurane $(1.5 \% \mathrm{v} / \mathrm{v})$ mixed with nitrous oxide and oxygen (45:55). The scalp was cleansed with Betadine and treated with local analgesic (bupivacaine). The skull was exposed and cleansed, and a metal head bolt was cemented to the back of the skull. Recording chambers were implanted in the skull above the tectal lobes to make them accessible for multiple experiments. The scalp was sutured, cleansed with Betadine, and treated locally with antibiotic ointment and analgesic. The owl recovered in a warm box before being returned to its aviary.

\section{Electrophysiology}

On the day of an experiment, the owl was anesthetized with $1.5 \%$ isoflurane mixed with nitrous oxide and oxygen and positioned in a padded plastic tube, and the head bolt was secured to the apparatus. The isoflurane was discontinued, and the owl was tranquilized with the nitrous oxide and oxygen mixture $(45 \% / 55 \%)$ for the duration of the experiment.

Action potentials were recorded extracellularly with epoxy-coated tungsten microelectrodes (10-12 M $\Omega$ at $1 \mathrm{kHz}$; FHC or A-M Systems). The raw signal was amplified and digitized with TDT hardware (RA-16; Tucker-Davis Technologies) controlled by custom scripts in MATLAB (The MathWorks) software. Spike times were collected from $400 \mathrm{~ms}$ before stimulus onset for a period of $1200 \mathrm{~ms}$.

Physiological landmarks were used to position the electrode in the Ipc. First, we positioned the electrode at the visual representation of contralateral $15^{\circ}$ azimuth and $-30^{\circ}$ elevation in the OT space map (Knudsen, 1982). Then, we moved the electrode medial 1.7-2.1 mm to hit the Ipc. On the way to the Ipc, units responded to auditory stimuli as the electrode passed through the central nucleus of the inferior colicullus. As the electrode entered the Ipc, units fired bursts of spikes spontaneously or in response to visual or auditory stimuli. In the final experiment, electrolytic lesions ( $10 \mu \mathrm{A}$ for $15 \mathrm{~s}$ ) were made at selected recording sites, and the locations of the lesions were subsequently reconstructed in transverse Nissl-stained sections.

\section{Visual stimuli}

Visual stimuli were generated with customized MATLAB software (courtesy of Joe Bergan, Harvard University, Cambridge, MA) and Psychophysics Toolbox extensions (Brainard, 1997; Pelli, 1997) and were presented (Mitsubishi XD300U projector) on a calibrated tangent screen $35 \mathrm{~cm}$ from the eyes. The owl was positioned so that the visual axes were in the horizontal plane aligned with $0^{\circ}$ azimuth and $0^{\circ}$ elevation of the screen; the eyes of barn owls are essentially stationary in the head. All locations are given in double pole coordinates of azimuth relative to the midsagittal plane and elevation relative to the visual plane. Visual stimuli were negative contrast (black) looming dots on a gray background. Looming dots were presented for $250 \mathrm{~ms}$ with 3-5 s interstimulus intervals. The radius of the looming dots started at $0.6^{\circ}$ and increased linearly for $250 \mathrm{~ms}$. Different loom speeds were achieved by changing the final dot sizes. Because Ipc units prefer small dot sizes (Asadollahi et al., 2010), the increased responses to faster loom speeds reflect preferences for faster speeds per se and not preferences for the larger final dot sizes of faster loom speeds. When two stimuli were presented simultaneously, both started and ended at the same time.

Spatial tuning curves. First, we estimated the RF by moving a full contrast dot manually around on the screen. Then, the computer presented single looming stimuli (at a constant loom speed) at seven equally spaced locations $\left(2-3^{\circ}\right.$ resolution $)$ in azimuth at a constant elevation across the RF. The stimulus locations were randomized and interleaved. On interleaved trials, we tested the effects of a competing stimulus located far outside the RF $\left(30^{\circ}\right.$ medial or lateral to the RF center) on the azimuthal tuning curve. For these tests, the $S_{\text {out }}$ stimulus was never positioned $>10^{\circ}$ ipsilateral (the space map represents locations up to $20^{\circ}$ ipsilateral) so that both the $S_{\text {in }}$ and $S_{\text {out }}$ stimuli were always represented on the same side of the brain. We refer to the stimulus inside the RF as the " $S_{\text {in }}$ " stimulus, and we refer to the competing stimulus outside the RF as the " $S_{\text {out }}$ " competitor. The $S_{\text {out }}$ was usually $4^{\circ} / \mathrm{s}$ stronger than the $S_{\text {in }}$ stimulus. Stimuli were repeated 10-25 times.

$S_{\text {in }}$ strength-response profiles. We evaluated the effects of a constant $S_{\text {out }}$ competitor on $S_{\text {in }}$ strength-response profiles by holding the location of the $S_{\text {in }}$ constant while changing its strength, with and without a distant $S_{\text {out }}$ competitor present. This test involved measuring two $S_{\text {in }}$ strengthresponse profiles: one profile measured responses to changes in the $S_{\text {in }}$ stimulus alone $\left[S_{\text {in }}\right.$ response profile $\left.\left(S_{\mathrm{i}} \mathrm{RP}\right)\right]$, the other measured responses to $\left[S_{\text {in }}+S_{\text {out }}\right]$ presented together $\left[\left[S_{\text {in }}+S_{\text {out }}\right]\right.$ response profile $\left.\left(S_{\mathrm{i}+\mathrm{o}} \mathrm{RP}\right)\right]$. For these tests, the $S_{\text {in }}$ stimulus was located at the center of the $\mathrm{RF}$ and its strength was varied across a range of $0-14^{\circ} / \mathrm{s}$, whereas the $S_{\text {out }}$ competitor was located $30^{\circ}$ to the side of the RF center and its strength was held constant (population average, $S_{\text {out }}=6.7 \%$ s; range, $6-10 \%$ ). These stimulus sets were interleaved and repeated 10-25 times.

To minimize habituation caused by stimulus repetition, we jittered the location of stimuli on subsequent presentations when locations were sampled repeatedly. Stimulus locations were jittered within a small region $\left( \pm 1.5^{\circ}\right.$ for $S_{\text {in }}$ and $\pm 2^{\circ}$ for $S_{\text {out }}$ ), centered on the desired location.

\section{Analysis of unit responses}

Responses to stimuli were analyzed in a response window from 100 to $250 \mathrm{~ms}$ after stimulus onset. Net responses were obtained by subtracting spontaneous discharge rates (counted in a window of 400-0 ms before stimulus onset and averaged across all stimulus presentations) from rates measured during the response window.

Spatial tuning curves. To test for any significant effect of an $S_{\text {out }}$ competitor on azimuthal tuning curves, responses to the $S_{\text {in }}$ alone presented from the three most central locations of the RF (three locations that elicited the greatest responses) were pooled and compared with the pooled responses to the same $S_{\text {in }}$ stimuli in the $\left[S_{\text {in }}+S_{\text {out }}\right]$ condition. $S_{\text {out }}$ was considered as having a significant effect on the responses when the two pooled response distributions were different at the $95 \%$ confidence level $(p<0.05, t$ test $)$

We quantified response suppression using the following formula:

$$
\% \text { suppression }=\frac{r_{\mathrm{i}}-r_{\mathrm{io}}}{r_{\mathrm{i}}} \times 100,
$$

where $r_{\mathrm{i}}$ is the average response to the $S_{\text {in }}$ alone, and $r_{\text {io }}$ is the average response to the $\left[S_{\text {in }}+S_{\text {out }}\right]$ condition

We estimated the center and width at half-maximum of azimuthal spatial tuning by fitting average responses with the following Gaussian function:

$$
r(x)=b+m \times e\left(-\frac{(x-c)^{2}}{\left(2 \times \sigma^{2}\right)}\right),
$$

where $r(x)$ is the average response at azimuth $x, c$ is the center of the RF, $\sigma$ is the $\mathrm{SD}$, which is $0.425 \times$ width at half-maximum, $m$ is the maximum response, and $b$ is the baseline activity. These parameters were used to evaluate the effects of an $S_{\text {out }}$ competitor on azimuthal tuning curves.

To assess subtractive or divisive effects of the $S_{\text {out }}$ competitor on responses to the $S_{\text {in }}$ stimulus, responses to the $\left[S_{\text {in }}+S_{\text {out }}\right]$ condition were plotted against responses to the corresponding $S_{\text {in }}$-alone condition and the data were fit by linear regression. We only included data for which the response to the $S_{\text {in }}$ alone was significantly above spontaneous activity (95\% confidence level). This was necessary to avoid forcing the $y$-intercept of the regression to zero, artifactually masking any subtractive effect. We calculated the slope and $y$-intercept of the best-fit regression line as estimates of the divisive and subtractive effects, respectively, of the $S_{\text {out }}$ competitor on $S_{\text {in }}$ responses.

$\mathrm{S}_{\text {in }}$ strength-response profiles. To test for any significant effect of the $S_{\text {out }}$ competitor on responses to the $S_{\text {in }}$ stimulus, all responses from the $S_{\mathrm{i}} R P$ that were significantly above spontaneous activity $(95 \%$ confidence level; $p<0.05, t$ test) were pooled and compared with the pooled responses from the $S_{\mathrm{i}+\mathrm{o}} \mathrm{RP}$. 
To quantify the effect of the $S_{\text {out }}$ competitor on $S_{\text {in }}$ strength-response profiles, we compared the $S_{\mathrm{i}} \mathrm{RPs}$ with the $S_{\mathrm{i}+\mathrm{o}} \mathrm{RPs}$. Average responses to $10-25$ stimulus repetitions were fitted with the following sigmoidal function:

$$
r(x)=L+\frac{S}{\left(1+e^{-m(x-d)}\right)}
$$

where $r(x)$ is the response at loom speed $x, L$ is the asymptotic minimum response; $S$ is the asymptotic maximum response; $d$ is the loom speed at the half-maximum response; and $m$ is the maximum slope. These four parameters, derived from sigmoidal fits to the $S_{\mathrm{i}} \mathrm{RPs}$, were compared with those derived from the sigmoidal fits to the $S_{\mathrm{i}+\mathrm{o}} \mathrm{RPs}$.

We used the following procedure to test for significant changes in $S_{\mathrm{i}} \mathrm{RP}$ slope ("transition range") and loom speed at half-maximum (" $50 \%$ point") caused by a competing stimulus. First, the data from the $S_{\mathrm{i}} \mathrm{RP}$ were best-fit with the sigmoidal function (Eq. 3). Then, the data from the $S_{\mathrm{i}+\mathrm{o}} \mathrm{RP}$ were best-fit with two sigmoidal functions: In the unconstrained condition (condition 1), all four parameters of the sigmoidal equation were treated as free parameters; in the constrained condition (condition 2 ), the value of the maximum slope (or the loom speed at half-maximum response) of the sigmoidal equation was fixed to the value derived from the $S_{\mathrm{i}} \mathrm{RP}$, whereas the other three parameters were treated as free parameters. The Akaike information criterion (AIC), which penalizes for the number of free parameters, was computed as a measure for goodness-offit for the two conditions. AIC values were compared with determine whether the unconstrained fit was of better quality (lower AIC value) than the constrained fit. If so, then the change in slope (or loom speed at half-maximum response) in the $S_{\text {io }} \mathrm{RP}$ was considered to be significant; if not, the change was considered to be not significant. By this procedure, a change in slope (or in loom speed at half-maximum response) was significant only if the value, based on the $S_{\mathrm{i}} \mathrm{RP}$, could not account for the data from the $S_{\mathrm{i}+\mathrm{o}} \mathrm{RP}$.

For statistical comparisons across populations, we first performed a Lilliefors test to assess the data for normal distribution. For data with normal distributions, we compared the data with $t$ tests or ANOVA and reported the results as means \pm SEM. Otherwise, we used nonparametric tests (Wilcoxon's rank test or Kruskal-Wallis test) and reported the results as medians and $95 \%$ confidence intervals [low high] of the median.

\section{Results}

\section{Effect of a competing stimulus on individual spatial tuning curves}

We tested the effects of a distant, competing stimulus on the spatial tuning curves of Ipc units. Looming dark dots, known to be highly effective stimuli for driving responses in the isthmotectal circuit, were used both as the stimulus inside the $\operatorname{RF}\left(S_{\text {in }}\right)$ and as the competing stimulus outside the $\mathrm{RF}\left(S_{\text {out }}\right)$. For these measurements, the $S_{\text {in }}$ stimulus was presented at different azimuths inside the RFs, whereas the $S_{\text {out }}$ competitor was located $\sim 30^{\circ}$ to the side of the RF center, well outside of the RF of the unit, and was always represented on the same side of the brain as the RF center (see Materials and Methods).

An example of a spatial tuning curve for a single unit, measured with a $4 \%$ s looming stimulus, is shown in Figure $1 \mathrm{~A}$. A Gaussian fit to these data (Fig. $1 C$, black line) yielded a RF centered at right $2.8^{\circ}$ azimuth with a width at half-maximum of $6.9^{\circ}$.

The effect of a distant, $S_{\text {out }}$ competitor on this spatial tuning curve was measured on interleaved trials by pairing the $4 \% \mathrm{~s}$ looming stimulus $\left(S_{\text {in }}\right)$ with an $8 \%$ s looming $S_{\text {out }}$ competitor presented at right $36^{\circ}$ (Fig. $1 B$ ). Despite being located far outside of the RF, the $S_{\text {out }}$ competitor exerted a clear inhibitory effect (Fig. $1 C)$. A Gaussian fit to the responses to the $\left[S_{\text {in }}+S_{\text {out }}\right]$ condition revealed that the RF was still centered at right $2.6^{\circ}$ azimuth and had a similar width at half-maximum of $6.4^{\circ}$. However, the maximum response was reduced by $25 \%$ (Fig. 1C). Thus, the com-
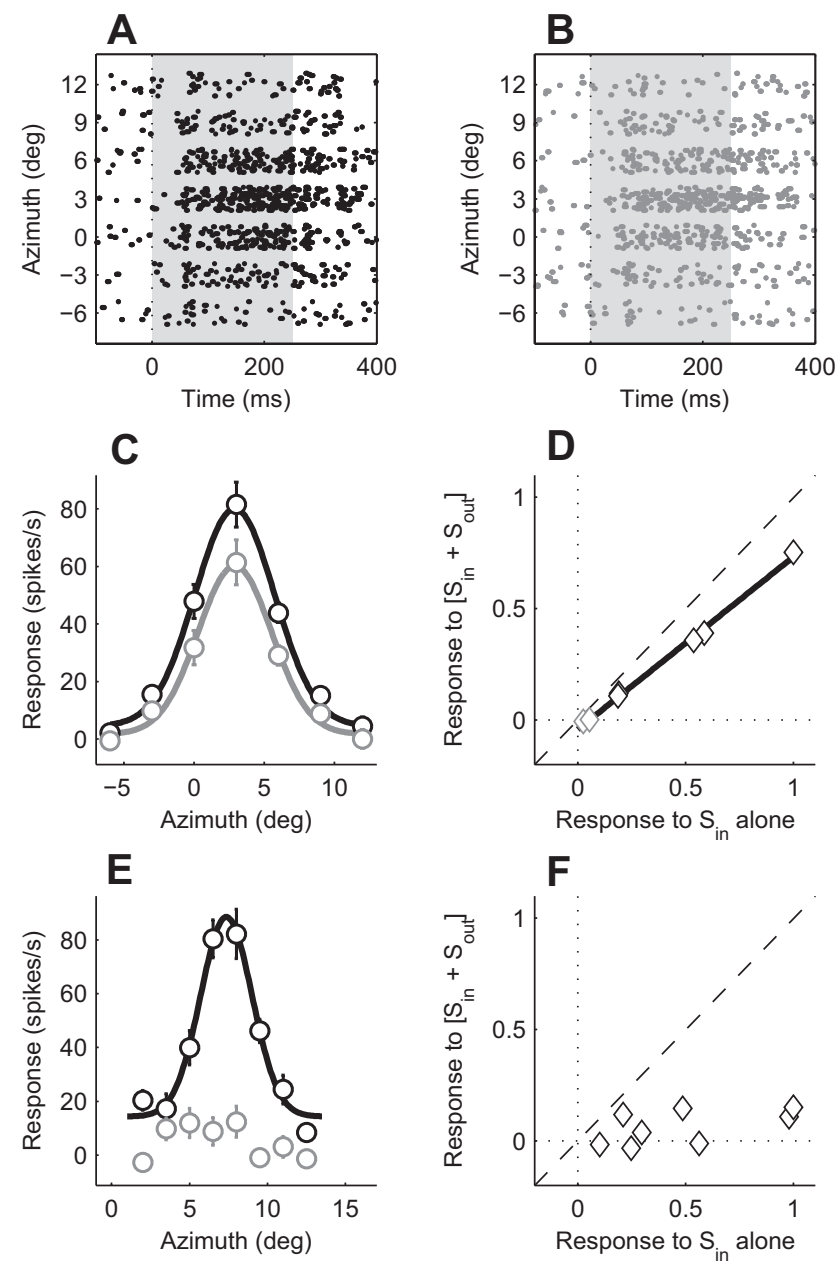

Figure 1. Divisive modulation of spatial tuning curve by a competing stimulus for representative Ipc units. $\boldsymbol{A}$, Raster representation of responses to the $S_{\text {in }}$ stimulus alone presented from different azimuths across the RF. Negative numbers indicate degrees to the left. $\boldsymbol{B}$, Raster representation of responses to the $S_{\text {in }}$ stimulus presented at different azimuths in presence of an $S_{\text {out }}$ competitor. $S_{\text {in }}$ loom speed, $4 \% \mathrm{~s} ; S_{\text {out }}$ loom speed, $8 \% \mathrm{~s}$. C, Spatial tuning curves derived from the data in $\boldsymbol{A}$ and $\boldsymbol{B}$. Black curve, Gaussian fit to the responses to $S_{\text {in }}$ alone; gray curve, Gaussian fit to the responses to $\left[S_{\text {in }}+S_{\text {out }}\right]$. Error bars show SEM. $D$, Normalized response to $\left[S_{\text {in }}+S_{\text {out }}\right]$ is plotted against the normalized response to the $S_{\text {in }}$ alone. The solid line shows the best linear fit to the data. Blue points, Nonsignificant responses to $S_{\text {in }}$ alone and excluded from linear fit. $\boldsymbol{E}, \boldsymbol{F}$, Responses from another unit presented in the same format as those for the unit in C and $\boldsymbol{D}$. For this unit, the $S_{\text {out }}$ competitor strongly suppressed responses to the $S_{\text {in }}$ stimulus.

petitor scaled down the spatial tuning curve without affecting its center or width.

To determine the extent to which the inhibitory effect of the competitor was divisive or subtractive, we plotted the responses to the paired $\left[S_{\text {in }}+S_{\text {out }}\right]$ condition as a function of the responses to the $S_{\text {in }}$-alone stimulus, for each location of the $S_{\text {in }}$ stimulus (Fig. $1 D$ ). Regression analysis revealed a linear relationship, with a slope of 0.77 and a $y$-intercept of -0.04 (robust regression, $p<$ 0.005 for slope; $p=0.16$ for intercept). To avoid forcing the $y$-intercept of the regression to zero, artifactually masking any subtractive effect, we only included data for which the response to the $S_{\text {in }}$ alone was significantly above spontaneous activity (see Materials and Methods) (Fig. $1 D$, black symbols). Such a linear relationship, with a slope $<1.0$, indicated that the $S_{\text {out }}$ stimulus exerted a divisive influence on responses to the $S_{\text {in }}$ stimulus. A $y$-intercept of zero indicated the absence of a subtractive inhibitory influence on $S_{\text {in }}$ responses. 
For another unit, the same strengths of $S_{\text {in }}$ and $S_{\text {out }}$ stimuli revealed a far stronger effect of the $S_{\text {out }}$ competitor on $S_{\text {in }}$ responses (Fig. $1 E, F$ ). In this case, the suppressive effect of the $S_{\text {out }}$ stimulus was so powerful that responses to the $\left[S_{\text {in }}+S_{\text {out }}\right]$ condition could not be fit with a Gaussian function (Fig. $1 E$, gray) and regression analysis could not be performed (Fig. $1 F$ ).

\section{Population analysis of the effect of a competitor on spatial tuning curves}

The strength of the suppressive effect of an $S_{\text {out }}$ competitor on spatial tuning curves was quantified as percentage suppression (Materials and Methods) for a population of 34 units. The locations of the RF centers for these units ranged from ipsilateral $10^{\circ}$ to contralateral $27^{\circ}$ in azimuth and from $-37^{\circ}$ to $+35^{\circ}$ in elevation. For these measurements, the $S_{\text {in }}$ stimulus had an average speed of $3.5 \%$; the $S_{\text {out }}$ competitor had an average speed of $7.6 \%$ and was always located $30^{\circ}$ to the side of the RF center, well outside of the RF.

The $S_{\text {out }}$ competitor nominally decreased responses to the $S_{\text {in }}$ stimulus for all 34 units. Across the entire population, percentage suppression ranged from 5.5 to $112.5 \%$ (suppression $>100 \%$ indicates suppression below spontaneous activity) with a median value of $29.2 \%$ (Fig. $2 A$ ). Although responses to the $S_{\text {in }}$ stimulus alone were always well fit by a Gaussian function $\left(R^{2}>0.75\right)$, responses to the $\left[S_{\text {in }}+S_{\text {out }}\right]$ condition were, for five units, suppressed so strongly that a Gaussian function could not be fit reliably (Figs. $1 E, F ; 2 A$, gray bars). Therefore, the data from these units were eliminated from additional analysis (the properties of these excluded units were otherwise not different from the remaining 29 less powerfully suppressed units, in terms of width of the tuning curves or spatial location of the RF: central vs peripheral; ipsilateral vs contralateral). Additional analyses were based on the data from the remaining 29 units (Fig. $2 A$, black and open bars). For eight of these units, response suppression by the $S_{\text {out }}$ competitor was weak and statistically not significant $(p<0.05, t$ test) (Fig. $2 A$, open bars). Inclusion or exclusion of the data from these eight units ( $n=29$ or 21 , respectively) did not change the results.

RF centers were unchanged by the presence of the $S_{\text {out }}$ competitor (average change, $0.13 \pm 0.11^{\circ} ; p=0.25 ; n=29 ; t$ test), and RF widths at half-maximum also remained unchanged $\left(S_{\text {in }}{ }^{-}\right.$ alone: average, $7.2 \pm 0.4^{\circ} \mathrm{SEM} ;\left[S_{\text {in }}+S_{\text {out }}\right]$ : average, $7.5 \pm 0.6^{\circ}$; $p=0.44 ; n=29 ; t$ test). These results held true also when the analysis was restricted to the units for which the $S_{\text {out }}$ competitor reduced responses significantly (RF centers: average change, $0.12 \pm 0.15^{\circ} ; p=0.42 ; t$ test; widths at half-maximum of the spatial tuning curves: $S_{\text {in }}$-alone, $7.4 \pm 0.5^{\circ} \mathrm{SEM} ;\left[S_{\text {in }}+S_{\text {out }}\right]$ : $7.6 \pm 0.7^{\circ} ; p=0.68 ; t$ test; $\left.n=21\right)$. Analysis of the population averages of the spatial tuning curves yielded similar results (Fig. $2 B$ ). The $S_{\text {out }}$ competitor reduced the maximum normalized response by $33 \%$, but it did not alter the width at half-maximum response (width at half-maximum for $S_{\text {in }}$ alone, $7.8^{\circ}$; and for the $\left[S_{\text {in }}+S_{\text {out }}\right]$ condition, $8.1^{\circ}$; average across 29 units).

The inhibitory effect of the $S_{\text {out }}$ competitor on spatial tuning curves was purely divisive. Responses to the $\left[S_{\text {in }}+S_{\text {out }}\right]$ condition were linearly related to responses to the $S_{\text {in }}$-alone condition. For individual units, the median slope was 0.73 , with a $95 \%$ confidence interval of [0.68 0.77] (Fig. $2 C)(p<0.001$, Wilcoxon's signed rank test in relation to $1 ; n=29)$ and the median $y$-intercept was $-0.01[-0.040 .02]$ (Fig. $2 D)(p=0.97$, Wilcoxon's signed rank test in relation to $0 ; n=29$ ). Restricting our analysis to the 21 units for which the $S_{\text {out }}$ competitor had a significant effect but did not completely suppress responses (Fig.
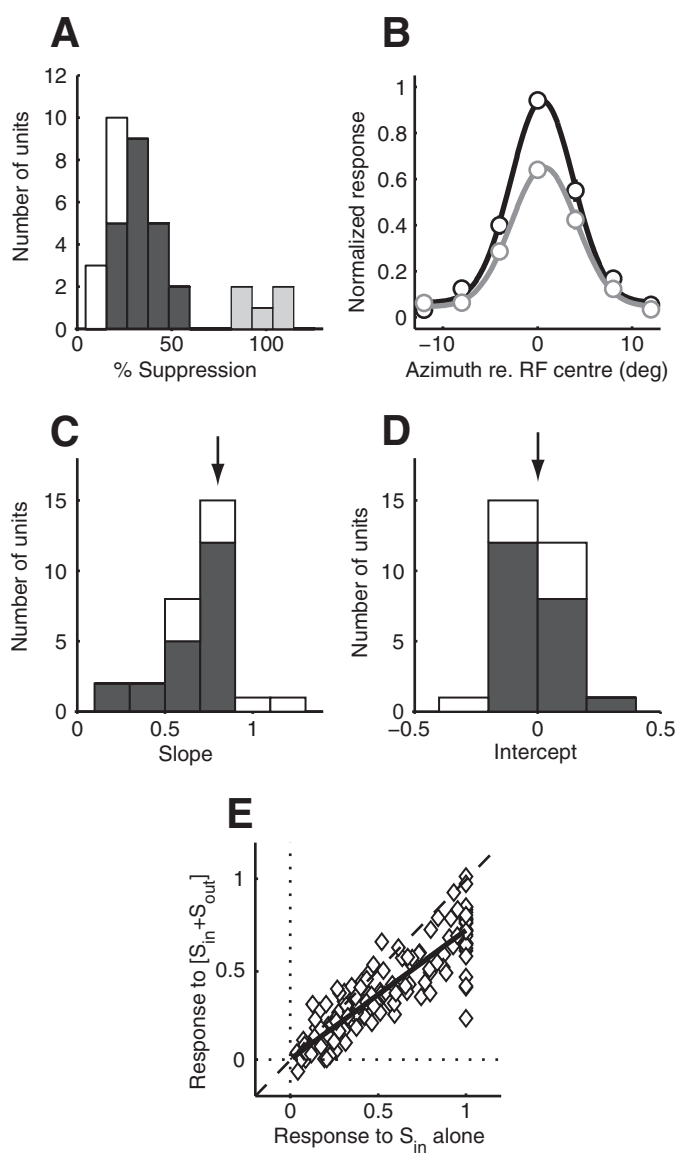

Figure 2. Population analysis of divisive modulation of spatial tuning curves by a competing stimulus. $A$, Distribution of percentage suppression of responses to $S_{\text {in }}$ alone by the $S_{\text {out }}$ competitor $(n=34)$. Gray bars, Five units that exhibited powerful suppression (these units were not be included in additional analyses); open bars, eight units for which the suppressive effect of the $S_{\text {out }}$ competitor was not significant $(p>0.05, t$ test). $\boldsymbol{B}$, Average of spatial tuning curves across 29 units measured using the $S_{\text {in }}$ stimulus without (black curve) and with (gray curve) the $S_{\text {out }}$ competitor stimulus. Error bars show SEM. C, Distribution of slopes of linear fits to the responses to $\left[S_{\text {in }}+S_{\text {out }}\right]$ versus the responses to $S_{\text {in }}$ alone for 29 units. The arrow indicates the mean. $\boldsymbol{D}$, Distribution of $y$-intercepts of linear fits to the responses to $\left[S_{\text {in }}+S_{\text {out }}\right]$ versus the responses to $S_{\text {in }}$ alone for 29 units. The arrow indicates the mean. $\boldsymbol{E}$, Normalized responses (154 data points from 29 units) to the simultaneous presentation of $\left[S_{\text {in }}+S_{\text {out }}\right]$ plotted against the normalized responses to $S_{\text {in }}$ alone. The thick line shows the linear regression of the data.

$2 A$, black bars) yielded similar results (median slope, $0.71[0.65$ 0.77 ]; $p<0.001$, Wilcoxon signed rank test in relation to 1 ; median $y$-intercept, $-0.01[-0.030 .01] ; p=0.88$, Wilcoxon's signed rank test in relation to 0). Pooled data from 29 units showed that spatial tuning curves were reduced divisively by the $S_{\text {out }}$ competitor: Responses to the $\left[S_{\text {in }}+S_{\text {out }}\right]$ condition were linearly related to responses to the $S_{\text {in }}$-alone condition with a slope of 0.71 and a $y$-intercept of zero (Fig. 2E) (regression analysis, $p<0.001$ for slope, $p=0.86$ for intercept; $n=29,154$ data points). Thus, the distant $S_{\text {out }}$ competitor suppressed responses to the RF stimulus divisively, by $\sim 30-40 \%$ for this particular combination of $S_{\text {in }}$ and $S_{\text {out }}$ strengths.

In summary, a competing stimulus exerted a strong inhibitory influence on spatial tuning curves without affecting their location or width. Based on results from a previous study (Asadollahi et al., 2010), this divisive inhibition operates globally across the entire space map. A heuristic schema of this competitive interaction is shown in Figure 3. In this schema, the $S_{\text {out }}$ competitor activates the inhibitory neurons that divisively modulate the responses of neurons representing the $S_{\text {in }}$ stimulus. 
Effects of a competing stimulus on $S_{\text {in }}$ strength-response profiles A second protocol for testing the effect of a competitor on stimulus-driven responses is to change the strength of the $S_{\text {in }}$ stimulus, while holding its location constant, with and without a distant $S_{\text {out }}$ competitor present. This protocol activated the circuitry that underlies stimulus competition differently from the first protocol (which changed excitatory drive by changing the location, rather than the strength, of the $S_{\text {in }}$ stimulus). This second protocol involved measuring two $S_{\text {in }}$ strength-response profiles: one profile described responses to changes in the $S_{\text {in }}$ stimulus alone $\left(S_{\mathrm{i}} \mathrm{RP}\right)$, and the other described responses to $\left[S_{\text {in }}+\right.$ $\left.S_{\text {out }}\right]$ presented together $\left(S_{\mathrm{i}+\mathrm{o}} \mathrm{RP}\right)$. For these tests, the $S_{\text {in }}$ stimulus was located at the center of the RF and its strength was varied across a range of $0-14 \%$, whereas the $S_{\text {out }}$ competitor was located $30^{\circ}$ to the side of the RF center, well outside of the $\mathrm{RF}$, and its strength was held constant (population average $S_{\text {out }}, 6.7 \%$ s; range, $6-10 \%$ s). In this test, the effect of the constant $S_{\text {out }}$ competitor was indicated by comparison of the $S_{\mathrm{i}+\mathrm{o}} \mathrm{RP}$ with the $S_{\mathrm{i}} \mathrm{RP}$.

Stimulus-response profiles for $\mathrm{S}_{i n}$-alone $\left(\mathrm{S}_{i} R P s\right)$

$S_{\mathrm{i}} \mathrm{RPs}$ were measured for 73 units (Fig. 4). Even a stationary dot (loom speed, $0 \%$ s), when centered in the RF, evoked responses that were significantly above baseline $(p<0.05$, Wilcoxon's signed rank test, in 65 of 73 units). For most units, responses increased with increasing $S_{\text {in }}$ loom speed ( 50 of 73 units; Spearman's rank correlation, $p<0.05$ ); the responses of these units usually plateaued at high loom speeds (Fig. $4 B, C$ ), although some units continued to increase their re-
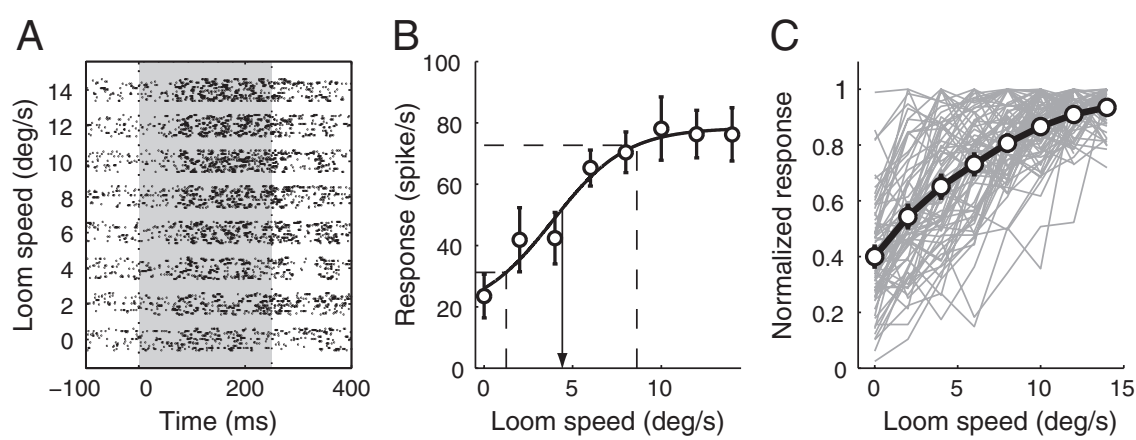

Figure 4. $\quad S_{\text {in }}$-alone strength-response profile $\left(S_{i} R P\right)$. $\boldsymbol{A}$, Raster representation of responses to a visual stimulus with variable loom speeds $\left(0-14^{\circ} / \mathrm{s}\right)$ presented at the center of the RF of an Ipc unit. $\boldsymbol{B}$, Average of responses in $\boldsymbol{A}$. Solid curve, The sigmoidal fit to the average responses; solid arrow, $50 \%$ point; dashed lines, lower and upper limits of the transition range. $C$, S RPs for Ipc units (gray lines). Black line, Average of $S_{i} R P$ s across Ipc units $(n=73)$.

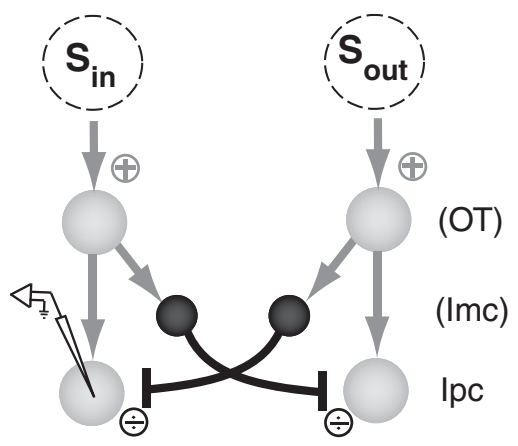

Figure 3. A heuristic schema of excitatory and inhibitory drives for two competing stimuli. The $S_{\text {in }}$ stimulus and the $S_{\text {out }}$ competitor, with respect to the recording site in the Ipc (indicated by the electrode symbol), are represented as two separate input channels. Blue, Excitation; black, divisive inhibition of Ipc output. For the Ipc circuit, we assume that the relevant excitatory drive originates in layer 10 of the $0 \mathrm{~T}$ and the inhibitory drive from the Imc. sponses to increasing speeds even up to

the highest loom speed tested (14\%) (Fig. 4C). The $S_{\mathrm{i}}$ RPs of these units were well fit with sigmoidal functions $\left(R^{2}>0.75 ; n=49\right)$ (Fig. $4 B$ ). From these sigmoidal fits, we derived the values of four parameters. The first parameter was the "minimum response": the response, based on the fit, for a loom speed of $0 \%$ s. Across the population, the average minimum response was $8.4 \pm 1.1$ spikes/s. The second parameter was the "maximum response": the fitted response for a loom speed of $14^{\circ}$ s. Across the population, the average maximum response was $44.4 \pm 2.9$ spikes/s. The third parameter was the "transition range": the range of loom speeds over which the responses changed from 10 to $90 \%$ of the total change in the response (Fig. $4 B$, dashed lines). Some neurons exhibited extremely narrow transition ranges, whereas others exhibited broad transition ranges that spanned almost the entire range of speeds that were tested. Across the population, the average transition range was $8.2 \pm 0.4 \%$. The fourth parameter was the " $50 \%$ point": the loom speed corresponding to the midpoint of the transition range (Fig. $4 B$, arrow). Across the population, the average $50 \%$ point was $5.8 \pm 0.3 \%$.

Stimulus-response profiles for the $\left[\mathrm{S}_{i n}+\mathrm{S}_{\text {out }}\right]$ condition $\left(\mathrm{S}_{i+o} R P S\right)$ An $S_{\text {out }}$ competitor suppressed responses to an $S_{\text {in }}$ stimulus (67 of total 73 units; $p<0.05$ ) (see Materials and Methods). However, unlike the effect expected from a constant divisive influence (Figs. $1 D, 3$ ), the suppression by the $S_{\text {out }}$ competitor was not a constant percentage of the response to the $S_{\text {in }}$-alone stimulus. Figure 5 shows examples of $S_{\mathrm{i}} \mathrm{RPs}$ and $S_{\mathrm{i}+\mathrm{o}} \mathrm{RPs}$ measured for four different units. In the first example, percentage suppression de- creased, rather than remaining constant, with increasing $S_{\text {in }}$ strength (Fig. $5 A$, right plot). In the second example, the transition range became narrower in the presence of the $S_{\text {out }}$ competitor (Fig. 5B, left plot). In the third example, the percentage suppression was not a linear function of $S_{\text {in }}$ strength and it decreased dramatically for high $S_{\text {in }}$ strengths (Fig. $5 C$ ). In the fourth example, although responses to the $S_{\text {in }}$-alone stimulus were similar across all $S_{\text {in }}$ strengths and were not well fit by a sigmoidal function (Fig. $5 D$, black circles), percentage suppression decreased with increasing $S_{\text {in }}$ strength (Fig. $5 D$, right plot), as in the first example (Fig. 5A).

Analysis of inhibition based on $\mathrm{S}_{i} R P s$ and $\mathrm{S}_{i+o} R P s$

Major categories of inhibition are as follows: divisive inhibition of output activity ("output division"; as in Fig. 2), divisive inhibition of input activity ("input division”), and subtractive inhibition. Each of these categories predicts different effects of a competitor on the $S_{\mathrm{i}} \mathrm{RP}$. Output division predicts a constant percentage suppression of responses across the range of $S_{\text {in }}$ strengths (Fig. 6A, right plot) and no changes in either the width of the transition range or the $50 \%$ point. Input division predicts a rightward shift of the $S_{\mathrm{i}} \mathrm{RP}$ (Fig. $6 \mathrm{~B}$, left plot), maximum percentage suppression for an intermediate range of $S_{\text {in }}$ strengths, either no change or an increase in the width of the transition range, and an increase in the $50 \%$ point (Ayaz and Chance, 2009). Subtractive inhibition predicts a downward shift of the $S_{\mathrm{i}} \mathrm{RP}$ (Fig. 6C), decreasing percentage suppression with increasing $S_{\text {in }}$ strength, and 
A

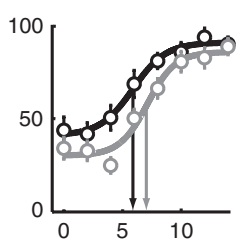

B

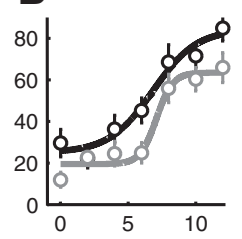

C

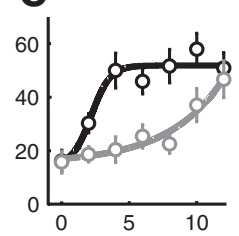

D

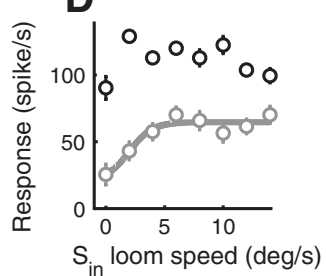

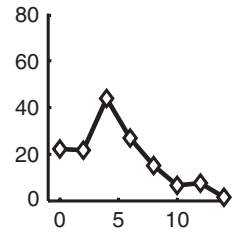
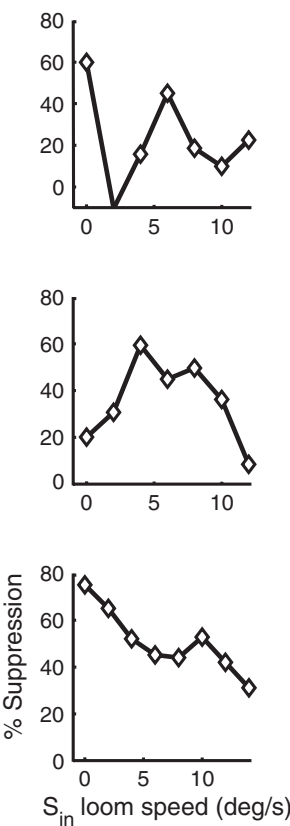

Figure 5. Effects of an $S_{\text {out }}$ competitor on $S_{i} R P s . A-D$, Four example units. Left panels, $S_{i} R P S$ (black) and $S_{\mathrm{i}+0} \mathrm{RPs}$ ( gray); the curves show the best sigmoidal fits to the data. Right panels, Percentage suppression plotted as a function of $S_{\text {in }}$ loom speed. The arrows (in $A$ ) designate $50 \%$ points. For $\boldsymbol{D}$, responses to $S_{\text {in }}$ alone (black circles) were not correlated with $S_{\text {in }}$ strength and were not well fit with a sigmoidal function.

no change in the width of the transition range or in the $50 \%$ point. We compared our data with these predictions.

We computed the percentage suppression of responses to the $S_{\text {in }}$ stimulus for different strengths of the $S_{\text {in }}$ stimulus (Fig. $7 A$ ). In contrast to the predictions of the major categories of divisive inhibition (Fig. $6 A, B$ ), the average percentage suppression decreased with increasing $S_{\text {in }}$ stimulus strength (Fig. 7A). Average percentage suppression decreased from $64 \%$ for an $S_{\text {in }}$ of $0 \%$, to $31 \%$ for an $S_{\text {in }}$ of $14 \%$ s $(p<0.001, t$ test; $n=73)$. These data demonstrate that percentage suppression does not remain constant with changes in $S_{\text {in }}$ strength.

Transition ranges tended to decrease in width in the presence of the $S_{\text {out }}$ competitor (Fig. $7 B$ ). For this analysis, we included those units, for which responses correlated with the $S_{\text {in }}$-alone loom speed and the $R^{2}$ of the best fits for both the $S_{\mathrm{i}} R P$ and the $S_{\text {io }} R P$ was $>0.75(n=47)$ (see Materials and Methods). Across 47 units, the average transition range for $S_{\mathrm{i}} \mathrm{RPs}$ was $7.8 \pm 0.4^{\circ} \% \mathrm{~s}$ and the average transition range for $S_{\mathrm{i}+\mathrm{o}} \mathrm{RPs}$ was $6.5 \pm 0.5 \%$ ( $t$ test, $p<0.01 ; n=47$ ) (Fig. $7 B$, all data). The significance of the difference between the transition range for the $S_{\mathrm{i}} \mathrm{RP}$ and the transition range for the $S_{\mathrm{io}} \mathrm{RP}$ was assessed for each unit (see Materials and Methods). For 21 (of 47) units, the transition range changed significantly in the presence of a competing stimulus. For these units, the average transition range for $S_{\mathrm{i}} \mathrm{RPs}$ was $7.7 \pm 0.6 \%$ and the average transition range for $S_{i+o} R P s$ was $5.1 \pm 0.8 \%$ s $(t$ test, $p<0.02$;
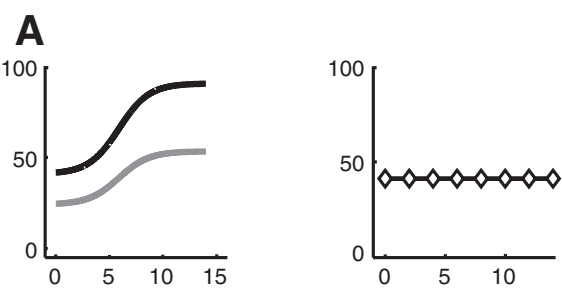

\section{B}
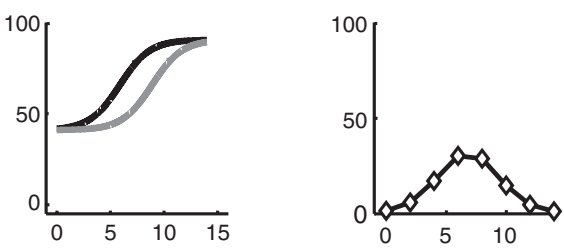

C
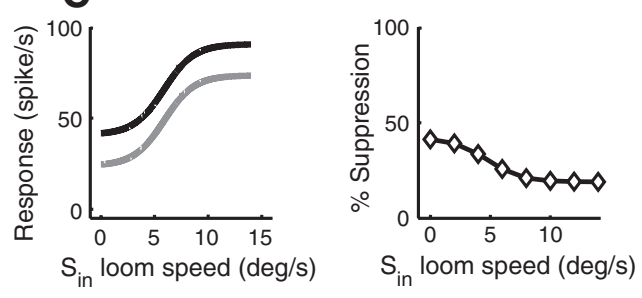

Figure 6. Changes in strength-response profiles predicted for different categories of inhibition, assuming constant inhibition because of the constant $S_{\text {out }}$ stimulus. Left panels, Black curves, $S_{\mathrm{i}} R P$ from the unit in Figure $5 A$; gray curves, hypothetical responses with inhibition. Right panels, Percentage suppression plotted as a function of $S_{\text {in }}$ strength. $A$, Output division, The $S_{i} R P$ was multiplied by 0.6 . $\boldsymbol{B}$, Input division, The $S_{i} R P$ was shifted rightward by $3 \%$ ( 0 lsen et al., 2010). C, Subtraction, Seventeen spikes were subtracted from each response.

$n=21$ ) (Fig. $7 B$, filled circles). The decrease in transition range width indicates an increased sensitivity to changes in $S_{\text {in }}$ strength in the presence of a competing stimulus. None of the major categories of inhibition (Fig. 6) predicts this effect.

Finally, the competitor tended to increase $50 \%$ points (Fig. $7 C$ ). The average $50 \%$ point for $S_{\mathrm{i}} \mathrm{RPs}$ (for those units for which responses correlated with the $S_{\text {in }}$-alone loom speed and the $R^{2}$ for both the $S_{\mathrm{i}} \mathrm{RP}$ and the $S_{\mathrm{io}} \mathrm{RP}$ was $\left.>0.75 ; n=47\right)$ was $5.9 \pm 0.3 \%$, whereas the average $50 \%$ point for $S_{\mathrm{i}+\mathrm{o}} \mathrm{RPs}$ was $7.3 \pm 0.3 \% \mathrm{~s}(t$ test, $p<0.001 ; n=47$; all circles) (Fig. $7 C$ ). The significance of the change in the $50 \%$ point was assessed for each unit (see Materials and Methods). For 30 (of 47) units, the $50 \%$ point for the $S_{\mathrm{i}+\mathrm{o}} \mathrm{RP}$ was significantly different from the $50 \%$ point for the $S_{\mathrm{i}} \mathrm{RP}$. The average $50 \%$ point for $S_{\mathrm{i}} \mathrm{RPs}$ was $5.8 \pm 0.4 \%$ and the average $50 \%$ point for $S_{i+o}$ RPs was $7.3 \pm 0.4^{\circ}$ s $(t$ test, $p<$ $0.001 ; n=30$ ) (Fig. $7 C$, filled circles). Among the major categories of inhibition, this particular result is only consistent with input division (Fig. 6C).

In addition, we found that, across the population of units, the magnitude of the increase in $50 \%$ points was predicted by the difference between the $S_{\mathrm{i}} \mathrm{RP} 50 \%$ point and the strength of the $S_{\text {out }}$ competitor (Fig. 7D) $(\rho=0.49 ; p<0.0001 ; n=47$; Spearman's rank correlation; Matlab corr function). As a result, the $S_{\text {out }}$ competitor caused a shift in the $50 \%$ point (point of maximum sensitivity to changes in $S_{\text {in }}$ strength) to a value that was on average not different from the strength of the $S_{\text {out }}$ competitor itself (mean \pm SEM difference, $-0.3 \pm 0.4 ; p=0.4, t$ test; $n=47$ ). These results are consistent with the idea that the strength of inhibition depends on the relative strengths of the $S_{\text {in }}$ and $S_{\text {out }}$ stimuli.

Indeed, in the measurements of stimulus-response profiles, evidence of output divisive inhibition by an $S_{\text {out }}$ competitor, as 

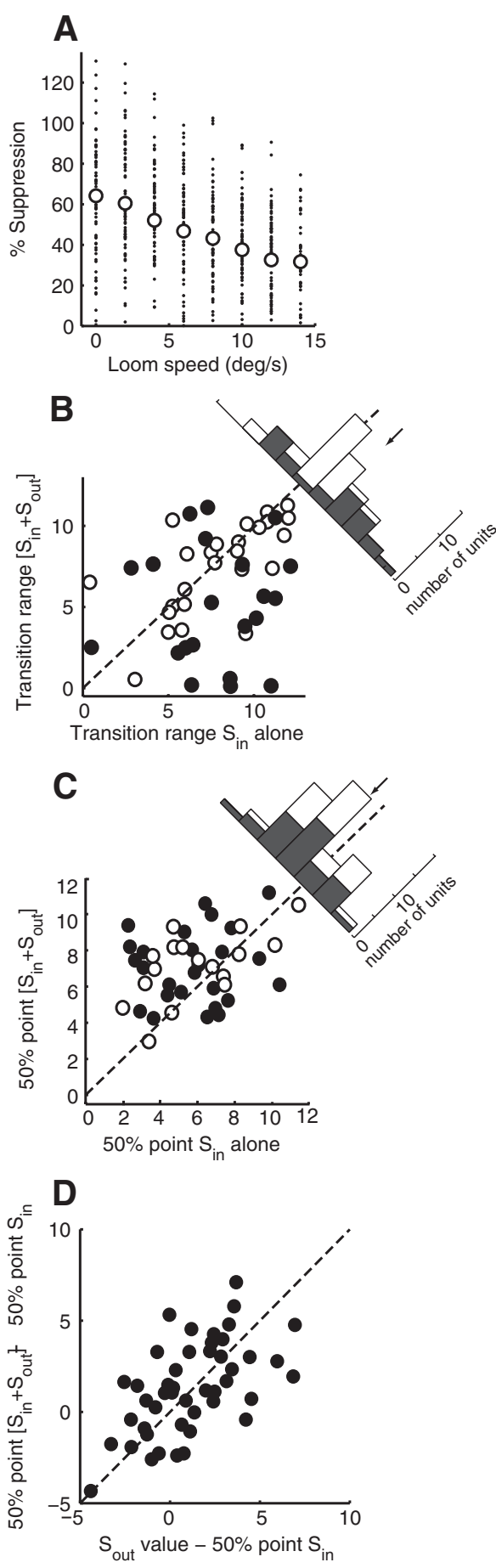

Figure 7. Effects of an $S_{\text {out }}$ competitor on $S_{i} R P s$. A, Percentage suppression for various $S_{\text {in }}$ strength for all units $(n=73)$. Dots, Percentage suppression for each unit; open circles, average percentage suppression across all units. $\boldsymbol{B}$, Transition range widths of $S_{i+0} R P S$ versus the transition range widths of $S_{i} R P s(n=47)$. Filled circles, Units that exhibited a significant change in transition range ( $n=21$ ) (see Materials and Methods). Histogram of change in transition range widths; arrow indicates mean across 47 units. C, The $50 \%$ points of $S_{i+0} R P s$ versus the $50 \%$ points of $S_{i} R P s(n=47)$. Filled circles, Units that exhibited a significant change in $50 \%$ points $(n=30)$ (see Materials and Methods). Histogram of change in $50 \%$ points; the arrow indicates mean across 47 units. $\boldsymbol{D}$, The change in the SRP $50 \%$ point ( $y$-axis) varied directly with the difference between the $50 \%$ point of the $S_{\mathrm{i}} \mathrm{RP}$ and the $S_{\text {out }}$ value ( $x$-axis) $(\rho=0.43 ; p<0.005$; Spearman's rank correlation; $n=47)$. Dashed lines, Equality line. depicted in Figure 3, was apparent only across the population when the relative strengths of the $S_{\text {in }}$ and $S_{\text {out }}$ stimuli were held constant (Fig. 8). When $S_{\text {in }}$ was $4 \%$ s weaker than $S_{\text {out }}$, population responses to the $S_{\text {in }}$ stimulus were inhibited by $\sim 68 \%$ (Fig. $8 \mathrm{~A}$ ) (intercept: 3.6 spikes/s; $p$ for intercept, 0.15 ; slope: $0.32 ; p$ for slope, $<0.0001$; regression analysis; $n=73$ ). When strength of the $S_{\text {in }}$ was equal to the strength of the $S_{\text {out }}$, population responses to the $S_{\text {in }}$ stimulus were inhibited by $44 \%$ (Fig. $8 B$ ) (intercept: -2.2 spikes/s; $p$ for intercept, 0.58 ; slope: 0.56 ; $p$ for slope, $<0.0001$; regression analysis; $n=73$ ), and when the $S_{\text {in }}$ stimulus was $4 \%$ stronger than the $S_{\text {out }}$ competitor, population responses to the $S_{\text {in }}$ stimulus were inhibited by $\sim 38 \%$ (Fig. $8 C$ ) (intercept: 6 spikes/s; $p$ for intercept, 2.1 ; slope: $0.62, p$ for slope, $<0.0001$; regression analysis; $n=73$ ). Based on these data, we propose that the effect of the $S_{\text {out }}$ competitor is indeed divisive, but that the strength of the divisive inhibition changes depending on the relative strengths of the $S_{\text {in }}$ and $S_{\text {out }}$ stimuli.

In summary, when the strength of an $S_{\text {in }}$ stimulus was systematically varied in the presence of a constant competing stimulus, responses to the $S_{\text {in }}$ stimulus were inhibited strongly when the $S_{\text {in }}$ stimulus was much weaker than the $S_{\text {out }}$ competitor, but were inhibited weakly when the $S_{\text {in }}$ stimulus was much stronger than the $S_{\text {out }}$ competitor. In addition, the $S_{\text {out }}$ competitor tended to narrow transition ranges and to shift $S_{\mathrm{i}} \mathrm{RP} 50 \%$ points toward the strength of the $S_{\text {out }}$ competitor. These properties deviate from those predicted for a constant divisive or subtractive influence from the constant competing stimulus (Fig. 6) and cannot be accounted for by a heuristic schema such as the one in Figure 3, even when implemented with a combination of divisive and subtractive influences.

\section{Effects of increasing competitor strength}

A third method for testing the effect of a competing stimulus on stimulus-driven responses is to hold the location and strength of the $S_{\text {in }}$ stimulus constant while systematically changing the strength of the $S_{\text {out }}$ competitor. We refer to responses measured with this test as a competitor strength-response profile (CRP). In this test, the $S_{\text {in }}$ stimulus was located at the center of the RF, and the $S_{\text {out }}$ competitor was located $30^{\circ}$ to the side of the RF center and its strength varied across a range of $0-14 \%$.

CRPs of Ipc units have been described previously (Asadollahi et al., 2010), and the following is a brief summary of the published findings. For $\sim 60 \%$ of Ipc units, CRPs revealed a gradual decline in responses to an $S_{\text {in }}$ stimulus as the strength of the $S_{\text {out }}$ competitor increased (Fig. $9 A$ ). For the remaining $40 \%$, responses to the $S_{\text {in }}$ stimulus remained strong until the $S_{\text {out }}$ competitor exceeded a critical value, beyond which responses to the $S_{\text {in }}$ stimulus were uniformly weak (Fig. 9B); such units were referred to as "switchlike units," and the value of the $S_{\text {out }}$ competitor that caused them to switch from strong to weak responses (the $50 \%$ point of the sigmoidal fit) was referred to as the "switch value." When a stronger $S_{\text {in }}$ stimulus was used to measure the CRP, switch values increased proportionately with the strength of the $S_{\text {in }}$ stimulus (Fig. 9C, arrows), demonstrating that the strength of inhibition by an $S_{\text {out }}$ competitor depended specifically on the relative strengths of the $S_{\text {in }}$ and $S_{\text {out }}$ stimuli, at least for switch-like units and perhaps for all Ipc units. Furthermore, CRP switch values were, on average, not different from the strength of the $S_{\text {in }}$ stimulus used to measure them. Similarly, we showed here that the $S_{\mathrm{i}+\mathrm{o}} \mathrm{RPs} 50 \%$ points were, on average, not different from the strength of the $S_{\text {out }}$ competitor used to measure them. These results indicate that Ipc units encode the strength of the $S_{\text {in }}$ stimulus relative to the strength of the $S_{\text {out }}$ competitor. 


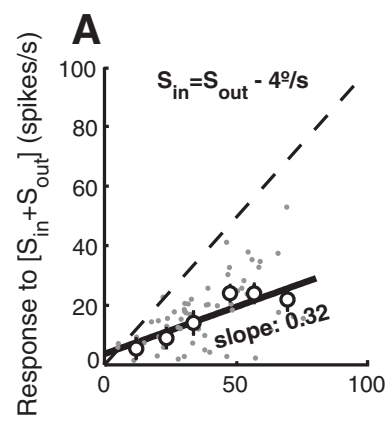

B

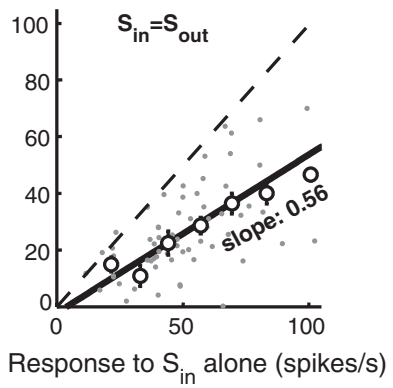

C

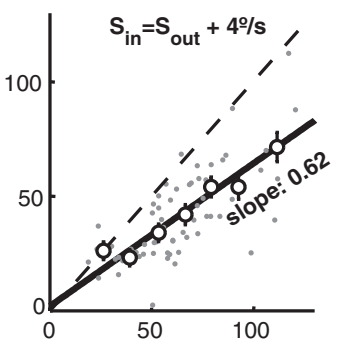

Figure 8. Relative strength of the $S_{\text {in }}$ stimulus and the $S_{\text {out }}$ competitor regulated the strength of divisive inhibition. Responses to the $\left[S_{\text {in }}+S_{\text {out }}\right]$ condition plotted against responses to the $S_{\text {in }}$ alone condition for a population of units $(n=47) . A, S_{\text {in }}$ was $4 \%$ s weaker than $S_{\text {out }} \boldsymbol{B}, S_{\text {in }}$ was equal to $S_{\text {out }} \boldsymbol{C}, S_{\text {in }}$ was $4 \%$ stronger than $S_{\text {out }}$. Dots, Data points; open circles, average of the binned data points; error bars, SD. Solid line, Best linear fit to the data. The slope indicates 1-strength of divisive inhibition. Dashed lines, Equality line.
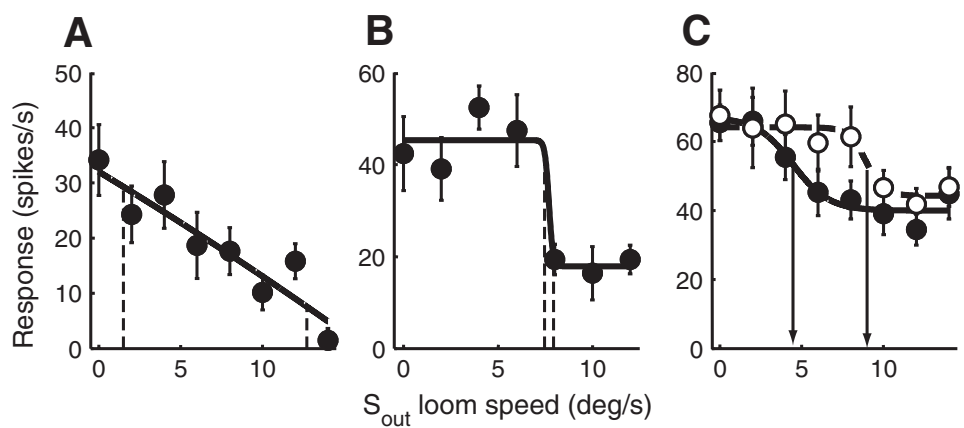

Figure 9. Effect of increasing strength of an $S_{\text {out }}$ competitor on responses to a constant $S_{\text {in }}$ stimulus (CRP). $A, A$ gradual CRP with a wide transition range (dashed lines). $\boldsymbol{B}, A$ switch-like (RP with a narrow transition range (dashed lines). $\boldsymbol{C}$, The switch point (vertical arrows) of the CRP increased with increasing $S_{\text {in }}$ stimulus strength. Open symbols, $S_{\text {in }}=4^{\circ} / \mathrm{s}$; filled symbols, $S_{\text {in }}=8^{\circ} / \mathrm{s}$. Error bars show mean $\pm S E M$ of responses. The lines are best sigmoidal fits to the data.
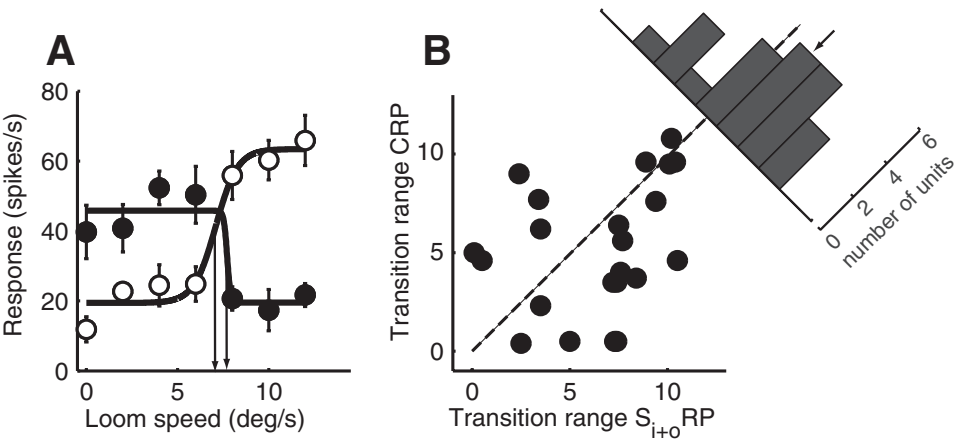

Figure 10. Comparison of $S_{i+0} R P s$ with CRPs. $A, S_{i+0} R P$ (open symbols) and CRP (filled symbols) for an Ipc unit. Error bars indicate mean \pm SEM of responses. The vertical arrows indicate the $50 \%$ point of the $S_{i+{ }_{0}} R P$ and the switch point of the CRP. The $x$-axis defines loom speed for the $S_{\text {in }}$ stimulus for the $S_{i+{ }_{0}} R P$ and loom speed for the $S_{\text {out }}$ stimulus for the CRP. $B$, Distribution of differences between the transition ranges derived from $S_{i+0} R P s$ and the transition ranges derived from CRPs. The transition ranges derived from $S_{i+0} R P s$ were weakly correlated with those derived from CRPs $(\rho=0.4 ; p=0.07 ;$ Spearman's rank correlation; $n=$ 22). Histogram, Difference between the transition ranges derived from the $S_{i+0} R P$ and the CRP; the arrow indicates median.

We tested this conclusion here by comparing the $S_{\mathrm{i}+\mathrm{o}} \mathrm{RP}$ (Fig. $10 \mathrm{~A}$, open circles) with the CRP (Fig. $10 \mathrm{~A}$, filled circles) for individual units. To make the $S_{\mathrm{i}+\mathrm{o}} \mathrm{RPs}$ and CRPs comparable, the strength of the constant $S_{\text {out }}$ competitor in the $S_{i+o} \mathrm{RP}$ protocol was set equal to the strength of the constant $S_{\text {in }}$ stimulus in the CRP protocol. The analysis was restricted to units for which responses to the $S_{i+o} R P$ and CRP protocols were well fit $\left(R^{2}>0.75\right)$ with a sigmoidal function. This analysis revealed that, although $S_{\mathrm{i}+{ }_{\mathrm{o}}} \mathrm{RP} 50 \%$ points and CRP switch points were not mutually correlated $(\rho=0.08 ; p=0.72$; Spearman's rank correlation; $n=22$ ), the difference between $S_{\mathrm{i}+\mathrm{o}} \mathrm{RP} 50 \%$ points and CRP switch points was not different from 0 across the population $\left(S_{\mathrm{i}+{ }_{\mathrm{o}}} \mathrm{RP} 50 \%\right.$ point - CRP switch point, $1.5 \pm 0.3^{\circ} \mathrm{s} ; p=0.07, t$ test $n=22$ ). In addition, the widths of transition ranges measured using the $S_{\mathrm{i}+\mathrm{o}} \mathrm{RP}$ protocol were, on average, not different from those measured using the CRP protocol (Fig. $10 B$ ): the median transition range for $S_{i+o}$ RPs was $7.4 \%$ [5.4 9.2] and for CRPs was $4.8 \% \mathrm{~s}$ [3.4 6.2] $(p=0.14$, Wilcoxon's sign rank test; $n=22)$.

\section{Discussion}

This study explored stimulus competition in the Ipc, a midbrain cholinergic structure that has been implicated in competitive stimulus selection and gaze control (Cui and Malpeli, 2003; Marín et al., 2007; Asadollahi et al., 2010). We found that a stimulus that is located far away from the RF of a unit divisively suppresses responses to a RF stimulus $\left(S_{\text {in }}\right)$ without altering RF sizes or locations. The suppression is readily accounted for by postulating that a globally projecting, divisive inhibitory network regulates responses in the Ipc. Moreover, by systematically changing the relative strengths of the $S_{\text {in }}$ and $S_{\text {out }}$ stimuli, we demonstrated that, for many units, the strength of the divisive inhibition changes markedly, depending on the relative strengths of the competing stimuli: inhibition is strong only when a competing stimulus is stronger than the RF stimulus. In this discussion, we compare inhibition in the Ipc with inhibition in typical sensory processing areas, and we propose that inhibition in the Ipc is special in that it involves feedback that regulates a network of globally projecting, divisive inhibitory neurons, a computational architecture that aids competitive stimulus selection.

Two major forms of inhibition that occur frequently in sensory structures are normalizing and surround inhibition. Normalizing inhibition serves to stabilize network activity and adjusts the sensitivity of neurons according to the average level of activity within a local pool of neurons, a computation that helps to avoid response saturation (Heeger, 1992; Carandini et al., 1997; Olsen et al., 2010). Indeed, for Ipc units that exhibit a gradual, progressive decline in responses with increasing strength of a competitor stimulus, global inhibition is performing a normalizing function. Normalizing inhibition is not selective for the value of the stimulus feature that is processed by the pool. For example, in the visual cortex, responses to grating stimuli presented at any nonpreferred orientation inside the RF divisively suppress orientation 
tuning curves, measured with a second grating (Tolhurst and Heeger, 1997; Busse et al., 2009; MacEvoy et al., 2009). In the visual cortex, the normalization process pools responses to all orientations for a restricted region in space (across the hypercolumn). In the Ipc, the normalization process pools activity across all space (across the entire structure).

A second major form of inhibition is surround inhibition. Stimuli located in the area immediately surrounding the RF often suppress responses to the RF stimulus (DeAngelis et al., 1994; Angelucci et al., 2002; Ozeki et al., 2004). The inhibition is usually strongest when the surrounding stimuli have the same feature properties as the preferred RF stimulus (Allman et al., 1985). Surround inhibition of this type sharpens the selectivity of neurons for the location and sometimes for the features of stimuli, and it is strongest for stimuli located close to the RF borders. Unlike surround inhibition, global competitive inhibition in the Ipc is not selective for stimulus features and the strength of the inhibition is similar across the visual field (Asadollahi et al., 2010).

Competitive surround inhibition in the Ipc is different from these forms of inhibition in two respects: it operates across the entire structure (it is global) and it is independent of stimulus feature and sensory modality (Asadollahi et al., 2010). Similar inhibition has been reported in the SC/OT of cats and owls (Rizzolatti et al., 1974; Mysore et al., 2010) and in the lateral intraparietal area of monkeys (Falkner et al., 2010), structures known to be involved in stimulus selection for gaze and spatial attention (McPeek and Keller, 2004; Bisley and Goldberg, 2010; Lovejoy and Krauzlis, 2010). In addition, computational models have invoked a similar form of inhibition to create salience maps for controlling gaze and spatial attention (Itti and Koch, 2001).

This study shows that the rule of competitive surround inhibition is unusual in yet another respect: the strength of inhibition is regulated specifically by the relative strengths of the stimuli. When the stimulus inside the RF is stronger, inhibition by the competing stimulus is weak and, for some units, not measurable (Fig. $5 A$, rightmost points). In contrast, when the stimulus inside the RF is weaker, inhibition by the competing stimulus is strong and can, for some units, completely suppress responses to the RF stimulus (Fig. 1E). This effect increases the steepness (narrows the transition ranges) of $S_{\mathrm{i}} \mathrm{RPs}$, reflecting an increase in the sensitivity of Ipc units to the relative strengths of competing stimuli specifically when the stimuli have similar strengths. In addition, a competing stimulus tends to cause a shift in the 50\% point of the $S_{\mathrm{i}} \mathrm{RP}$ toward the strength of the competitor. This competitive rule enhances the representation of the strongest (most salient) stimulus.

Competitive inhibition in the Ipc cannot be accounted for by constant mutual inhibition. Deviation from constant mutual inhibition is conspicuous in CRPs, in which the strength of an RF stimulus is kept constant while the strength of an $S_{\text {out }}$ competitor varies. A gradual increase in the strength of a competing stimulus causes a gradual increase in inhibition (gradual responses) for some units, but for many other units, it causes an abrupt increase in inhibition as the competitor becomes the stronger stimulus (switch-like responses) (Asadollahi et al., 2010). Whereas gradual
B
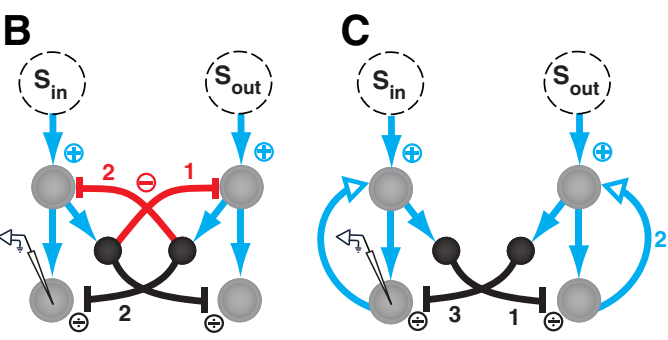

responses might reflect response normalization, switch-like responses must serve a different function. Moreover, increasing the strength of the RF stimulus causes a proportional increase in the switch value for switch-like responses (Fig. 9C) (Asadollahi et al., 2010). With constant divisive inhibition, increasing the strength of the RF stimulus would scale the magnitude of the CRP, but it would not shift the switch value.

Adding a feedback element to the heuristic schema in Figure 3 yields a network architecture that could account qualitatively for the competitive interactions that we observed in the Ipc (Ayaz and Chance, 2009). This feedback element could be implemented in several different ways (Fig. 11). The networks presented in Figure 11 each show only two of the many spatial channels that exist in the Ipc. Global, divisive inhibition is represented by the direct inhibitory pathway from the channel that encodes the $S_{\text {out }}$ stimulus to the channel that encodes the $S_{\text {in }}$ stimulus (Fig. 11, black). The strength of that inhibition could be regulated by mutual, direct inhibitory connections between the components that mediate the divisive inhibition (Fig. $11 A$, red). Alternatively, the inhibitory component being driven by the $S_{\text {in }}$ spatial channel could inhibit the excitatory input to the competing $S_{\text {out }}$ spatial channel (Fig. $11 B$, red 1). This inhibition of excitatory input to the competing channel would, in turn, suppress the competitive inhibition of the $S_{\text {in }}$ spatial channel (Fig. $11 B$, red 2 and black 2). Alternatively, the inhibitory component being driven by the $S_{\text {in }}$ spatial channel inhibits the Ipc component in the competing $S_{\text {out }}$ channel (Fig. 11C, black 1) and that Ipc component could send a feedback connection to the input layer, the function of which is to increase excitatory responses in that spatial channel (Fig. 11C, blue open arrow 2). The suppression of feedback excitation in the $S_{\text {out }}$ channel would decrease excitatory drive in that channel and would, in turn, decrease the competitive inhibition of the $S_{\text {in }}$ channel (Fig. 11C, black 3). All of these architectural motifs result in competitive regulation of global, divisive inhibition. When the strengths of all connections are equal, these network architectures will cause the inhibition of responses to the $S_{\text {in }}$ stimulus, in the presence of the $S_{\text {out }}$ stimulus, to be weak when $S_{\text {in }}>S_{\text {out }}$ and to be strong when $S_{\text {in }}<S_{\text {out }}$, as was observed in the Ipc.

All of the architectural motifs shown in Figure 11 appear to exist in the isthmotectal network. The isthmic complex includes a globally projecting GABAergic nucleus, the Imc, located just below the OT in the lateral tegmentum (Sereno and Ulinski, 1987; Wang et al., 2004). Neurons in the Imc receive a topographic input from an intermediate layer (layer 10) of the OT. They project forward to the Ipc (Fig. 11, black) and they project back to the 
OT to all portions of the space map except the portion that provides their input (Fig. $11 B$, red); the potential importance of this pathway to competitive interactions has been modeled (Lai et al., 2011). In addition, Ipc units project back topographically to the retinal input layers of the OT and, presumably, facilitate visual responses in those layers (Fig. $11 C$, blue open arrows). Furthermore, single-cell labeling of Imc neurons reveals axon collaterals that terminate locally within the Imc (Wang et al., 2004), and these GABAergic axon terminals are likely to inhibit other Imc neurons (Fig. $11 \mathrm{~A}$, red). However, none of these circuits has yet to be shown to operate in the manner depicted in Figure 11, and additional research is required to determine whether any of these motifs is critical to the competitive interactions that take place in the Ipc.

Regardless of which, if any, of these motifs is critical, the architecture of the Imc circuit is clearly suited for distributing inhibition throughout the Ipc and the OT space maps. Moreover, inactivation of the Imc greatly reduces competitive inhibition in the Ipc (Marín et al., 2007). We hypothesize, therefore, that competitive interactions in the Ipc result from the regulation of global divisive inhibition mediated by the Imc.

\section{References}

Allman J, Miezin F, McGuinness E (1985) Stimulus specific responses from beyond the classical receptive field: neurophysiological mechanisms for local-global comparisons in visual neurons. Annu Rev Neurosci 8:407-430.

Angelucci A, Levitt JB, Walton EJ, Hupe JM, Bullier J, Lund JS (2002) Circuits for local and global signal integration in primary visual cortex. J Neurosci 22:8633-8646.

Asadollahi A, Mysore SP, Knudsen EI (2010) Stimulus-driven competition in a cholinergic midbrain nucleus. Nat Neurosci 13:889-895.

Ayaz A, Chance FS (2009) Gain modulation of neuronal responses by subtractive and divisive mechanisms of inhibition. J Neurophysiol 101: 958-968.

Bisley JW, Goldberg ME (2010) Attention, intention, and priority in the parietal lobe. Annu Rev Neurosci 33:1-21.

Brainard DH (1997) The Psychophysics Toolbox. Spat Vis 10:433-436.

Busse L, Wade AR, Carandini M (2009) Representation of concurrent stimuli by population activity in visual cortex. Neuron 64:931-942.

Carandini M, Heeger DJ, Movshon JA (1997) Linearity and normalization in simple cells of the macaque primary visual cortex. J Neurosci 17: 8621-8644.

Carello CD, Krauzlis RJ (2004) Manipulating intent: evidence for a causal role of the superior colliculus in target selection. Neuron 43:575-583.

Cui H, Malpeli JG (2003) Activity in the parabigeminal nucleus during eye movements directed at moving and stationary targets. J Neurophysiol 89:3128-3142.

DeAngelis GC, Freeman RD, Ohzawa I (1994) Length and width tuning of neurons in the cat's primary visual cortex. J Neurophysiol 71:347-374.

Falkner AL, Krishna BS, Goldberg ME (2010) Surround suppression sharpens the priority map in the lateral intraparietal area. J Neurosci 30:12787-12797.
Fecteau JH, Munoz DP (2006) Salience, relevance, and firing: a priority map for target selection. Trends Cogn Sci 10:382-390.

Heeger DJ (1992) Normalization of cell responses in cat striate cortex. Vis Neurosci 9:181-197.

Itti L, Koch C (2001) Computational modelling of visual attention. Nat Rev Neurosci 2:194-203.

Knudsen EI (1982) Auditory and visual maps of space in the optic tectum of the owl. J Neurosci 2:1177-1194.

Lai D, Brandt S, Luksch H, Wessel R (2011) Recurrent antitopographic inhibition mediates competitive stimulus selection in an attention network. J Neurophysiol 105:793-805.

Lovejoy LP, Krauzlis RJ (2010) Inactivation of primate superior colliculus impairs covert selection of signals for perceptual judgments. Nat Neurosci 13:261-266.

MacEvoy SP, Tucker TR, Fitzpatrick D (2009) A precise form of divisive suppression supports population coding in the primary visual cortex. Nat Neurosci 12:637-645.

Maczko KA, Knudsen PF, Knudsen EI (2006) Auditory and visual space maps in the cholinergic nucleus isthmi pars parvocellularis of the barn owl. J Neurosci 26:12799-12806.

Marín G, Salas C, Sentis E, Rojas X, Letelier JC, Mpodozis J (2007) A cholinergic gating mechanism controlled by competitive interactions in the optic tectum of the pigeon. J Neurosci 27:8112-8121.

McPeek RM, Keller EL (2004) Deficits in saccade target selection after inactivation of superior colliculus. Nat Neurosci 7:757-763.

Mysore SP, Asadollahi A, Knudsen EI (2010) Global inhibition and stimulus competition in the owl optic tectum. J Neurosci 30:1727-1738.

Olsen SR, Bhandawat V, Wilson RI (2010) Divisive normalization in olfactory population codes. Neuron 66:287-299.

Ozeki H, Sadakane O, Akasaki T, Naito T, Shimegi S, Sato H (2004) Relationship between excitation and inhibition underlying size tuning and contextual response modulation in the cat primary visual cortex. J Neurosci 24:1428-1438.

Parkhurst D, Law K, Niebur E (2002) Modeling the role of salience in the allocation of overt visual attention. Vision Res 42:107-123.

Pelli DG (1997) The VideoToolbox software for visual psychophysics: transforming numbers into movies. Spat Vis 10:437-442.

Rizzolatti G, Camarda R, Grupp LA, Pisa M (1974) Inhibitory effect of remote visual stimuli on visual responses of cat superior colliculus: spatial and temporal factors. J Neurophysiol 37:1262-1275.

Sereno MI, Ulinski PS (1987) Caudal topographic nucleus isthmi and the rostral nontopographic nucleus isthmi in the turtle, Pseudemys scripta. J Comp Neurol 261:319-346.

Tolhurst DJ, Heeger DJ (1997) Contrast normalization and a linear model for the directional selectivity of simple cells in cat striate cortex. Vis Neurosci 14:19-25.

Wang SR (2003) The nucleus isthmi and dual modulation of the receptive field of tectal neurons in non-mammals. Brain Res Brain Res Rev 41: $13-25$.

Wang Y, Major DE, Karten HJ (2004) Morphology and connections of nucleus isthmi pars magnocellularis in chicks (Gallus gallus). J Comp Neurol 469:275-297.

Wang Y, Luksch H, Brecha NC, Karten HJ (2006) Columnar projections from the cholinergic nucleus isthmi to the optic tectum in chicks (Gallus gallus): a possible substrate for synchronizing tectal channels. J Comp Neurol 494:7-35. 